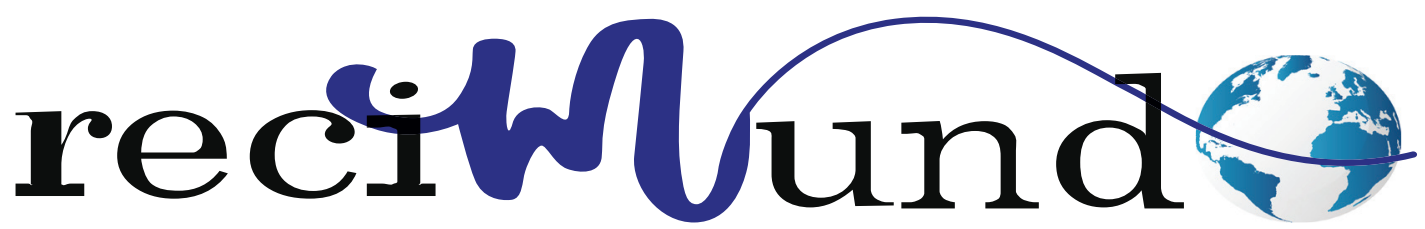

Revista Científica Mundo de la Investigación y el Conocimiento

DOI: 10.26820/recimundo/5.(4).oct.2021.163-171

URL: https://recimundo.com/index.php/es/article/view/1315

EDITORIAL: Saberes del Conocimiento

REVISTA: RECIMUNDO

ISSN: 2588-073X

TIPO DE INVESTIGACIÓN: Artículo de revisión

CÓDIGO UNESCO: 32 Ciencias Médicas

PAGINAS: 163-171

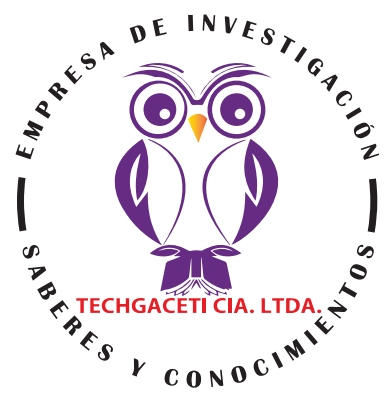

\title{
Infarto agudo al miocardio sin lesiones coronarias obstructivas (MINOCA)
}

Acute myocardial infarction without obstructive coronary lesions (MINOCA) Infarto agudo do miocárdio sem lesões coronárias obstrutivas (MINOCA) Satya Lucia Soria Bajaña'; Mario Fernando De La Pared Ramírez; Kristel Delgado Lazo; Nataly Gardenia Ortega Romero ${ }^{4}$

RECIBIDO: 02/09/2021 ACEPTADO: 20/09/2021 PUBLICADO: 30/10/2021

1. Médico, Investigador Independiente; Guayaquil, Ecuador; satya_soria8812@hotmail.com; (D) https://orcid.org/0000-00020626-327X

2. Médico, Investigador Independiente; Guayaquil, Ecuador; dmario_11@hotmail.com; (ID) https://orcid.org/0000-0002-1915438X

3. Médico, Investigador Independiente; Guayaquil, Ecuador; kristeldelgado90@gmail.com; (DD https://orcid.org/0000-00016943-0007

4. Médico, Investigador Independiente; Guayaquil, Ecuador; natalyor_@hotmail.com; (D) https://orcid.org/0000-0002-32205129

CORRESPONDENCIA

Satya Lucia Soria Bajaña

satya_soria8812@hotmail.com

Guayaquil, Ecuador

() RECIMUNDO; Editorial Saberes del Conocimiento, 2021 


\section{RESUMEN}

El infarto de miocardio con arterias coronarias no obstruidas (MINOCA, por sus siglas en inglés) ha ganado importancia en los últimos 20 años debido a la dilucidación de etiologías fisiopatológicas diferentes de las causas obstructivas del flujo coronario (obstrucción definida como estenosis $<50 \%$ ). Diversos estudios han evidenciado una prevalencia que varía entre el $6 \%$ y el 25\%, la cual llega a ser más alta en las mujeres (10-25\%). Entre las múltiples causas de MINOCA existen diferentes grupos de alteraciones en la reactividad coronaria: las epicárdicas y las microvasculares, entre las cuales se incluyen espasmo coronario, disrupción de la placa aterosclerótica y disección coronaria, enfermedad de takotsubo, miocarditis y tromboembolia coronaria. El uso combinado de tomografía de coherencia óptica y resonancia magnética incrementa el diagnóstico del mecanismo causal en los Minoca, con un diagnóstico final en la mayoría de los casos de infarto de miocardio. El tratamiento de MINOCA se basa en pruebas limitadas y no existen ensayos controlados, aleatorizados y prospectivos. Los medicamentos (aspirina, estatinas, betabloqueantes, clopidogrel, inhibidores de la enzima convertidora de angiotensina/bloqueadores de los receptores de angiotensina) deben considerarse sobre la base del mecanismo subyacente de MINOCA en cada individuo. El enfoque general del presente trabajo es dar a conocer las características, factores de riesgo, métodos de diagnóstico y tratamiento del MINOCA. Los resultados se obtuvieron mediante el desarrollo de una investigación de tipo bibliográfica, delimitada a una metodología de revisión, que permite concluir que a pesar del creciente desarrollo de investigación en esta área, se requieren estudios que permitan obtener conocimiento más preciso del mecanismo fisiopatológico subyacente, lo que permitirá un uso racional y ajustado de la terapéutica disponible y la exploración de nuevos fármacos, tanto para el tratamiento sintomático como para la evolución pronóstica.

Palabras clave: Minoca, infarto, miocardio, lesiones coronarias, riesgo cardiovascular.

\section{ABSTRACT}

Myocardial infarction with unobstructed coronary arteries (MINOCA) has gained importance in the last 20 years due to the elucidation of pathophysiological etiologies other than obstructive causes of coronary flow (obstruction defined as stenosis $<50 \%)$. Various studies have shown a prevalence that varies between $6 \%$ and $25 \%$, which is higher in women (10-25\%). Among the multiple causes of MINOCA there are different groups of alterations in coronary reactivity: epicardial and microvascular, including coronary spasm, atherosclerotic plaque disruption and coronary dissection, takotsubo disease, myocarditis, and coronary thromboembolism. The combined use of optical coherence tomography and magnetic resonance imaging increases the diagnosis of the causal mechanism in Minoca, with a final diagnosis in most cases of myocardial infarction. The treatment of MINOCA is based on limited evidence and there are no prospective, randomized, controlled trials. Medications (aspirin, statins, beta-blockers, clopidogrel, angiotensin converting enzyme inhibitors / angiotensin receptor blockers) should be considered based on the underlying mechanism of MINOCA in each individual. The general approach of this work is to present the characteristics, risk factors, methods of diagnosis and treatment of MINOCA. The results were obtained through the development of a bibliographic type research, limited to a review methodology, which allows to conclude that despite the growing development of research in this area, studies are required that allow obtaining more precise knowledge of the underlying pathophysiological mechanism, This will allow a rational and adjusted use of available therapeutics and the exploration of new drugs, both for symptomatic treatment and for prognostic evolution.

Keywords: Minoca, infarction, myocardium, coronary lesions, cardiovascular risk.

\section{RESUMO}

O infarto do miocárdio com artérias coronárias desobstruídas (MINOCA) ganhou importância nos últimos 20 anos devido à elucidação de outras etiologias fisiopatológicas que não as causas obstrutivas do fluxo coronariano (obstrução definida como estenose $<50 \%$ ). Vários estudos mostram uma prevalência que varia entre $6 \%$ e $25 \%$, sendo mais elevada nas mulheres (10-25\%). Entre as múltiplas causas do MINOCA existem diferentes grupos de alterações na reatividade coronariana: epicárdica e microvascular, incluindo espasmo coronariano, ruptura da placa aterosclerótica e dissecção coronariana, doença de takotsubo, miocardite e tromboembolismo coronariano. O uso combinado de tomografia de coerência óptica e ressonância magnética aumenta o diagnóstico do mecanismo causal em Minoca, com diagnóstico final na maioria dos casos de infarto do miocárdio. O tratamento do MINOCA é baseado em evidências limitadas e não há estudos prospectivos, randomizados e controlados. Os medicamentos (aspirina, estatinas, betabloqueadores, clopidogrel, inibidores da enzima de conversão da angiotensina / bloqueadores do receptor da angiotensina) devem ser considerados com base no mecanismo subjacente do MINOCA em cada indivíduo. A abordagem geral deste trabalho é apresentar as características, fatores de risco, métodos de diagnóstico e tratamento do MINOCA. Os resultados foram obtidos através do desenvolvimento de uma pesquisa do tipo bibliográfico, limitada a uma metodologia de revisão, que permite concluir que apesar do crescente desenvolvimento das pesquisas nesta área, são necessários estudos que permitam obter um conhecimento mais preciso do mecanismo fisiopatológico subjacente. permitirá o uso racional e adequado das terapêuticas disponíveis e a exploração de novos fármacos, tanto para o tratamento sintomático como para a evolução prognóstica.

Palavras-chave: Minoca, infarto, miocárdio, lesões coronárias, risco cardiovascular. 


\section{Introducción}

El infarto agudo de miocardio (IAM) es la principal causa de muerte en el mundo, siendo la obstrucción coronaria aterosclerótica el hallazgo más frecuente. Sin embargo, Matsudo et al. (2019) indica que:

\begin{abstract}
El 6\% de los pacientes no presenta lesiones angiográficamente significativas, definidas por obstrucción de la luz vascular mayor al $50 \%$. Estos casos se han definido bajo el término MINOCA. Suelen ocurrir en mujeres jóvenes, con factores de riesgo cardiovascular, elevación de biomarcadores cardíacos e infradesnivel del segmento ST en el electrocardiograma. (Matsudo et al. 2019).
\end{abstract}

Entre las múltiples causas del infarto agudo al miocardio sin lesiones coronarias obstructivas (MINOCA por sus siglas en inglés) que se han evidenciado en los estudios Cleves et al. (2021) establece que:

Existen diferentes grupos de alteraciones en la reactividad coronaria: las epicárdicas y las microvasculares, entre las cuales se incluyen espasmo coronario, disrupción de la placa aterosclerótica y disección coronaria, enfermedad de takotsubo, miocarditis y tromboembolia coronaria. ( $p$. 186).

La identificación etiológica es esencial para definir el tratamiento adecuado a la fisiopatología y al pronóstico del paciente. En este sentido Ache et al. (2020) indican que:

Las herramientas diagnósticas iniciales del MINOCA incluyen la historia clínica, ECG, troponinas, ecocardiografía transtorácica, angiografía coronaria con test de va- sorreactividad y ventriculografía izquierda. El motivo de consulta suele ser dolor torácico y se manifiesta en el ECG más frecuentemente sin elevación del segmento ST. El valor de corte angiográfico de obstrucción coronaria $<50 \%$ es arbitrario y deben reconocerse factores limitantes: existe variabilidad inter e intraobservador de la estimación angiográfica de las estenosis, la trombosis es un proceso dinámico y la presencia de estenosis coronarias significativas no descarta de forma absoluta la posibilidad de MINOCA. (Ache et al. 2020).

En cuanto al tratamiento Vidal (2017) destaca que los efectos beneficiosos a largo plazo en pacientes con esta patología se obtienen con el tratamiento con estatinas y IECA/ARAll, se observa una tendencia hacia un efecto positivo para los betabloqueantes y un efecto neutro de la DAG.

El objetivo de este trabajo investigativo, desarrollado bajo un diseño documental, se centra en realizar una revisión a la literatura científica disponible, que principalmente aborde el infarto agudo al miocardio sin lesiones coronarias obstructivas (MINOCA), sus características, principales factores de riesgo, métodos de diagnóstico y tratamiento, todo ello con la finalidad de proporcionar un material actualizado que defina dichos aspectos y que sea de utilidad no solo para el personal de salud, sino también para el público en general.

\section{Materiales y Métodos}

El presente trabajo de investigación, enmarcado en una metodología de revisión, está orientado a la construcción de un material bibliográfico actualizado, enfocado en dar a conocer los criterios vigentes del infarto agudo al miocardio sin lesiones coronarias obstructivas (MINOCA). 
Entre los portales web consultados destacan: Sociedad Española de Cardiología, Scielo, entre otras. Como términos de búsqueda se utilizaron las expresiones "Minoca" y "Infarto agudo al miocardio sin lesiones coronarias obstructivas", y se aplicaron criterios de selección tales como: idioma español e inglés; publicación entre 2015 y 2021 (ambos inclusive); acceso completo y abierto; en el área de salud y medicina; estudios referidos a humanos; tipo de bibliografía, manuales médicos, guías clínicas, ensayos clínicos, estudios o reportes de casos, boletines y/o folletos de instituciones oficiales o privadas de excelente trayectoria en el área de la salud, medicina o científico académica, y demás, monografías y otros documentos que, a criterio propio, mostraran información de interés en base a la observación de la evidencia científica referida en sus contenidos. Este proceso arrojó resultados que en promedio oscilaron entre 6 y 58 enlaces a fuentes de información bibliográficas.

De igual manera fueron adelantados otras sondeos menores sin considerar la aplicación de cualquier otro criterio de descarte, ya que se requirió encontrar información complementaria que independientemente de su origen o época de publicación, es considerada igualmente importante ajustada y de relevancia para este tema. Es a partir de entonces que se procedió con la lectura crítica y análisis interpretativo de un pilar de información recopilado a lo largo de la investigación, que también fue adoptada como evidencia. Resultando todo este proceso en la selección de los elementos más sustanciales y significativos de las diferentes fuentes bibliográficas que fundamentan el razonamiento aquí expuesto.

\section{Resultados}

El infarto de miocardio con arterias coronarias no obstruidas (MINOCA, por sus siglas en inglés) ha ganado importancia en los últimos 20 años debido a la dilucidación de etiologías fisiopatológicas diferentes de las causas obstructivas del flujo coronario (obstrucción definida como estenosis < 50\%). (Cleves et al. 2021)

Diversos estudios han evidenciado una prevalencia que varía entre el $6 \%$ y el $25 \%$, la cual llega a ser más alta en las mujeres (10-25\%). Entre las múltiples causas que se han evidenciado en los estudios se encuentran diferentes grupos de alteraciones en la reactividad coronaria: las epicárdicas y las microvasculares. (p. 186).

El término MINOCA fue introducido por primera vez por John Beltrame en 2013 para reemplazar la terminología anterior de infarto de miocardio con coronarias normales (MINCA) que incluía solo a pacientes sin aterosclerosis de los vasos epicárdicos y no a pacientes con estenosis angiográficas entre $1 \%$ y $50 \%$. (Pérez, 2021).

Por su parte Lozano (2021) señala que la prevalencia de infartos de miocardio sin obstrucción coronaria en una revisión sistemática de estudios oscila entre 1-14\% ( $6 \%$ de media). Más frecuente en mujeres (10-25\%) frente a los hombres (6-10\%). La edad media de presentación es de 55 años. La mortalidad intrahospitalaria supone $0,9 \%$ y a los 12 meses del $4,7 \%$.

Entre las características que definen esta patología Pérez (2021) destaca las siguientes:

- Los pacientes tienen peor supervivencia y mayor riesgo de eventos repetidos comparados con individuos sanos. La tasa de eventos cardíacos adversos mayores en seguimiento a 4 años después de MINOCA es del 25\%, y se ha informado una mortalidad anual del $11 \%$.

- MINOCA se informa en el 6-15\% de los pacientes con IAM, se observa generalmente en pacientes relativamente jóvenes con menor prevalencia de factores 
de riesgo cardiovascular tradicionales, y es más frecuente en mujeres y en etnias negras, maoríes e hispanas

- Según la cuarta definición universal de IAM y como resultado de las pruebas de diagnóstico posteriores a la $\mathrm{ACl}$, un MINOCA puede terminar con un diagnóstico de IAM de tipo 1 y tipo 2 debido a los mecanismos de isquemia miocárdica que son de naturaleza aterosclerótica y no aterosclerótica.

- Cuando no se encuentra una lesión con un grado de estenosis del $50 \%$ o mayor en un vaso epicárdico mayor en la arteriografía, se debe realizar una reevaluación cuidadosa o incluso una ACI repetida para determinar ya sea una lesión culpable leve, una rama lateral ocluída en el origen o una lesión que se pasó por alto.

- En el HARP-MINOCA, un estudio prospectivo y multicéntrico de 145 mujeres con un diagnóstico final de MINOCA, los sitios calificaron la angiografía coronaria como normal en el $53,8 \%$ de las pacientes, mientras que el laboratorio central angiográfico informó un cateterismo normal (es decir, sin estenosis del $10 \%$ o más) en solo el 3,4\%. El pronóstico depende en gran medida de la causa subyacente, que sigue siendo desconocida en una proporción considerable de pacientes.

Los pacientes diagnosticados de MINOCA sufren un evento cardiaco incluido dentro de lo síndromes coronarios agudos, por lo que es importante determinar el papel que realizan los factores de riesgo cardiovasculares en este tipo de eventos. Entre los que Lozano (2021) destaca:

- Angina Vasoespástica: Entidad clínica caracterizada por la hiperreactividad de las arterias coronarias a los estímulos vasoconstrictores. Reducción del vaso $>75 \%$ tras un test de provocación + signos/síntomas, que afecta a pacientes varones y asiáticos. Tratamiento de elección con Nitratos/Ca-agonistas.

- Placa aterosclerótica excéntrica: placas que crecen hacia el exterior de los vasos, manteniendo la luz del vaso permeable. Son placas vulnerables, con gran cantidad de lípidos que se rompen fácilmente y embolizan a distal.

- Disección coronaria: Hemorragia intramural que forma un falso lumen provocando una disección y compresión de la luz arterial verdadera y como consecuencia un infarto agudo al miocardio (IAM).

- Takotsubo: Disfunción ventricular izquierda con hipocinesia o acinesia en segmentos medio-apicales con coronarias. Afecta a mujeres tras un evento emocional importante. Naturaleza transitoria y recurrente.

- Miocarditis: infiltrado inflamatorio del miocardio con necrosis y/o degeneración de los miocitos adyacentes. Su tratamiento no precisa medidas antiisquémicas.

- Embolia coronaria: Formación de un coagulo, ya sea en las arterias coronarias o en otra localización, que se desprende y migra hasta la microvasculatura coronaria o bien a una de las arterias coronarias epicárdicas. Los factores de riesgo son: ACXFA, endocarditis, mixoma, prótesis valvulares, estado protrombótico. El tratamiento consiste en la anticoagulación.

Según Matsudo et al. (2019) el diagnóstico de esta patología se propone como un método de trabajo exhaustivo con el fin de identificar o excluir posibles etiologías y para ello el uso de la cardiorresonancia se ha convertido en la herramienta clave para orientar el diagnóstico.

Por su parte Zuluaga \& Cano (2018) describen que así como en la insuficiencia cardiaca aguda, el MINOCA debe ser un diagnóstico de trabajo del cual debe buscarse su etiología. 
Para esto, inicialmente se deben obtener datos importantes de la historia clínica para guiar la búsqueda: antecedentes de enfermedad viral reciente, enfermedades autoinmunes, trauma psicológico/emocional previo al episodio, antecedentes familiares de trombofilias, medicamentos, uso de drogas psicoactivas y características del dolor torácico. Posteriormente, hay que recordar que la elevación de troponinas no es específica de enfermedad coronaria, por lo cual se deberían excluir causas potenciales no isquémicas de su elevación como: enfermedad renal, miocarditis, sepsis, tromboembolia pulmonar, trauma, quemaduras, entre otras. La mayoría de estas condiciones son fáciles de excluir en el escenario clínico. (pág. 375-376).

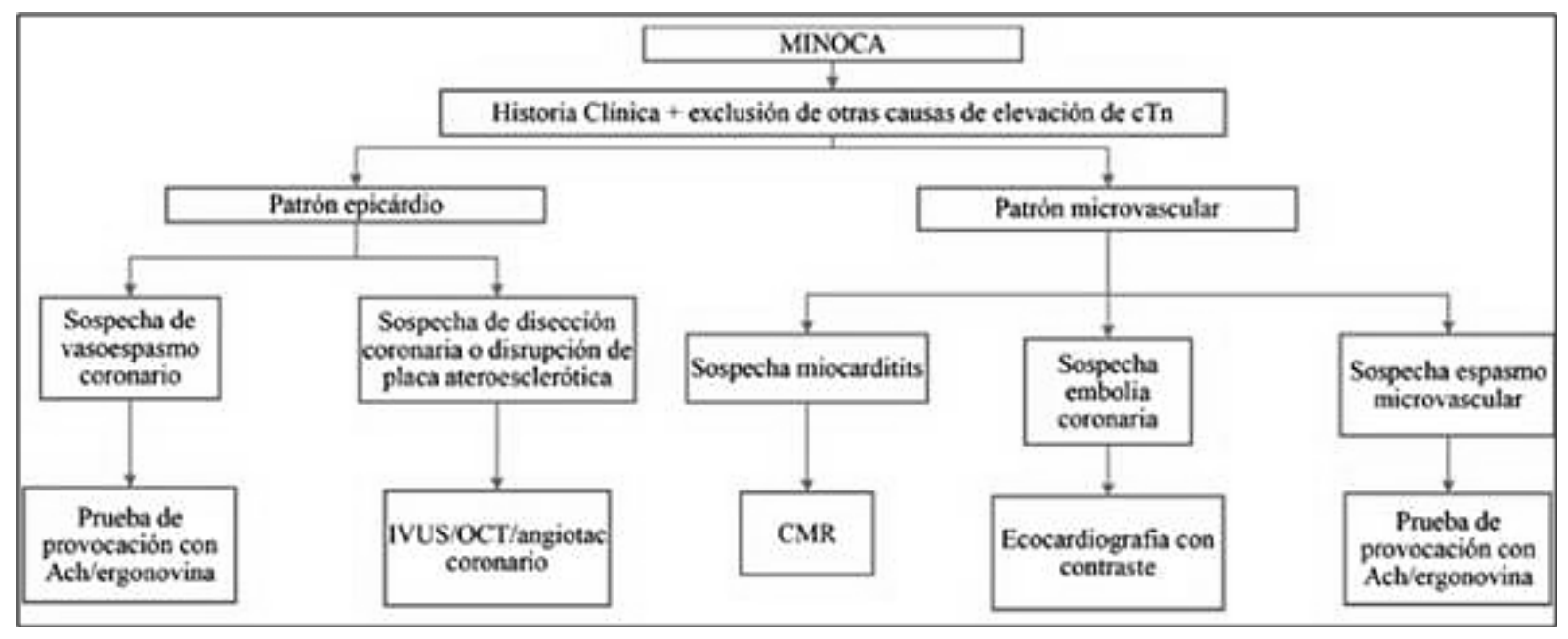

Figura 1. Algoritmo Diagnóstico.

Nota. Recuperado de: Infarto agudo de miocardio sin enfermedad coronaria ateroesclerótica obstructiva. Zuluaga \& Cano (2018). latreia. 31(4). Pág. 376. Recuperado de: http:// www.scielo.org.co/pdf/iat/v31n4/0121-0793-iat-31-04-00371.pdf

Para Corros (2021) llegar al diagnóstico final de esta entidad no siempre es posible. En este sentido, la RMC ha demostrado jugar un papel fundamental permitiendo diferenciar la afectación miocárdica de origen isquémico de otras entidades como la miocarditis o miocardiopatías de otras etiologías.

El uso combinado de tomografía de coherencia óptica y resonancia magnética incrementa el diagnóstico del mecanismo causal en los Minoca, con un diagnóstico final en la mayoría de los casos de infarto de miocardio (Corros , 2021).

En estudio realizado por Díaz (2020) analizó el rendimiento de la tomografía de co- herencia óptica intravascular (OCT), capaz de identificar diferentes cambios morfológicos de las placas coronarias conocidas causas de MINOCA, y de la cardiorresonancia magnética (CRM), convertida en técnica gold standard para la detección de infarto agudo de miocardio (IAM) en el contexto de MINOCA.

La CRM es recomendada como clave en el estudio de MINOCA, ya que proporciona confirmación y localización del IAM. En este estudio prospectivo se muestra que lesiones de alto riesgo valoradas por OCT constituyen evidencia directa de aterosclerosis coronaria como factor causante del daño miocárdico detectado en la CRM de los pacientes con MINOCA, por lo que la realización de ambos (tras exclusión del 
espasmo coronario mediante provocación con metilergonovina) puede permitir un diagnóstico definitivo, aumentando la conformidad en el tratamiento recomendado. (Díaz, 2020).

De acuerdo a lo indicado por Ache et al. (2020) en los pacientes diagnosticados con esta patología, el tratamiento no es la revascularización coronaria, lo que conlleva una dificultad terapéutica al no existir guías estandarizadas para su abordaje.

Un análisis por puntaje de propensión de 9.136 pacientes con MINOCA del regis- tro SWEDEHEART, comprobó que el tratamiento con estatinas, con agentes moduladores del sistema renina-angiotensina, y posiblemente con betabloqueantes, disminuye el riesgo de eventos adversos cardiovasculares mayores, no así el tratamiento con agentes antiplaquetarios. Sin embargo, hasta la fecha no existen ensayos clínicos randomizados sobre tratamiento del MINOCA y dada la heterogeneidad de los pacientes incluidos bajo este término tan amplio, el consenso actual es que debe realizarse un abordaje individualizado según el mecanismo fisiopatológico involucrado. (Ache et al. 2020).

Tabla 1. Principales mecanismos del MINOCA con su potencial tratamiento.

\begin{tabular}{|c|c|c|}
\hline Mecamismo fisiopatoligion & Imestigaciôn dinica & Temapia potencial \\
\hline $\begin{array}{l}\text { Disrupción de placa: } \\
\text { - Erosión de placa } \\
\text { - Ruptura de placa }\end{array}$ & $\begin{array}{l}\text { Tomografia de coherencia óptica } \\
\text { - Ultrasonido intravascular }\end{array}$ & - Estatinas \\
\hline $\begin{array}{l}\text { Tromboembolismo coronario: } \\
\text { - Trombosis } \\
\text { - Embolismo }\end{array}$ & $\begin{array}{l}\text { - Tomografía de coherencia óptica } \\
\text { - Trombofilia congénita } \\
\text { - Trombofilia adquirida }\end{array}$ & $\begin{array}{l}\text { - Agentes antiplaquetarios } \\
\text { - Anticoagulantes }\end{array}$ \\
\hline $\begin{array}{l}\text { Disfunción vasomotora } \\
\text { coronaria: } \\
\text { - Espasmo coronario } \\
\text {. Disfunción microvascular }\end{array}$ & $\begin{array}{l}\text { - Test de espasmo coronario } \\
\text { - Reserva de flujo fraccional } \\
\text { - Indices de resistencia } \\
\text { microvascular }\end{array}$ & $\begin{array}{l}\text { - Bloqueadores de los canales de } \\
\text { calcio }\end{array}$ \\
\hline
\end{tabular}

Nota. Infarto agudo de miocardio sin lesiones coronarias obstructivas - MINOCA: un enigma para el cardiólogo clínico. Ache. (2020). Revista Uruguaya de Cardiología 35(1). Recuperado de: http://www.scielo.edu.uy/scielo.php?pid=S1688-04202020000100202\&script=sci_arttext

En este mismo sentido, Cohen et al. (2021) indica que el tratamiento se inicia al ingreso y se relaciona con el diagnóstico final. $Y$ recomienda el siguiente esquema:

- Antiplaquetarios: Los pacientes con angor y elevación de troponina son tratados inicialmente con terapia antiplaquetaria simple o doble desde el ingreso (aspirina y clopidogrel o ticagrelor) y anticoagulados con heparina. De acuerdo a los resultados de la co- ronariografía, podrían considerarse dos posibilidades:

- Si se realizó ecografía coronaria intravascular/tomografía de coherencia óptica (IVUS/OCT) y se detectaron placas accidentadas, se recomienda mantener la doble terapia antiagregante durante un año. Si no se detectaron placas: suspender.

- Si no se realizó IVUS/OCT se evaluarán diagnósticos diferenciales. Si no puede descartarse un evento de placa se

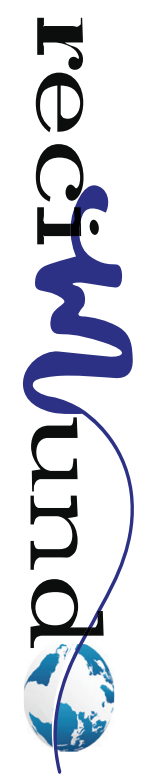


recomienda continuar con doble antiagregación durante un año.

- Anti-isquémicos: En los pacientes con vasoespasmo, se recomienda el tratamiento con bloqueantes cálcicos. Deberían evitarse los beta-bloqueantes, excepto los que tienen acción vasodilatadora asociada o son donadores de óxido nítrico.

- Tratamiento al alta: Considerando el rol esencial de la aterosclerosis en la modalidad clásica de la enfermedad, y trasladando la evidencia consolidada de sus beneficios, se considera que los dos objetivos terapéuticos primordiales son el tratamiento anti aterosclerótico y anti isquémico.

- Estatinas: existen evidencias de que altas dosis de estatinas mejoran la disfunción endotelial. Se demostró reducción del volumen y estabilización de la placa. Se considera que existe un efecto de clase por lo que no puede recomendarse una estatina en especial.

- Inhibición del eje neurohormonal: se recomienda el uso de IECA / ARA II. Tanto Ios IECA como los ARAll mejoran la reserva de flujo y favorecen la regresión de la fibrosis periarteriolar.

- Aspirina: no está claro su rol en esta población, por lo que se recomienda solamente en pacientes con alguna sospecha o evidencia de accidente de placa.

- Beta bloqueantes: se sugieren los de última generación por sus propiedades vasodilatadoras, metabólicas (carvedilol), o por ser dadores de óxido nítrico (nevibolol).

- Bloqueantes cálcicos: cuando se sospecha vasoespasmo de la microcirculación, se sugieren como primera elección.

- La terapia combinada entre beta-bloqueantes y dihidropiridínicos, suele ser beneficiosa por sus efectos farmacológicos complementarios, aunque se requiere un cuidado especial para evitar trastornos de conducción.

- Ivabradine: podría ser de utilidad por su efecto sobre la FC manteniendo la vasodilatación coronaria durante el ejercicio y mejorando la circulación colateral.

- Nitritos: útiles en episodios de angor de reposo y prolongados. Se pueden asociar a bloqueantes cálcicos. Sin embrago, se han descripto casos de efecto paradojal con exacerbación de síntomas.

- Trimetazidina: en pacientes con dolor refractario al tratamiento antiisquémico, se asocia a menor número de episodios isquémicos.

Por su parte Tamis et al. (2019), presenta un estuido donde indica que el tratamiento de MINOCA se basa en pruebas limitadas y no existen ensayos controlados, aleatorizados y prospectivos.

Los medicamentos (aspirina, estatinas, betabloqueantes, clopidogrel, inhibidores de la enzima convertidora de angiotensina / bloqueadores de los receptores de angiotensina) deben considerarse sobre la base del mecanismo subyacente de MINOCA en cada individuo. Si existe alguna evidencia de aterosclerosis, los factores de riesgo de EAC modificables deben tratarse de manera agresiva. El vasoespasmo coronario se trata mejor con bloqueadores de los canales de calcio y los beneficios de los nitratos de acción prolongada son menos claros. (Tamis et al. 2019).

\section{Conclusión}

El infarto de miocardio sin obstrucción coronaria (MINOCA) es un síndrome con múltiples causas potenciales por lo que establecer una etiología precisa es fundamental para el manejo y el pronóstico.

La falta de ensayos clínicos sobre el diagnóstico y tratamiento, el desconocimiento de la fisiopatología, la heterogeneidad de las etiologías y la falta de consensos de las sociedades de cardiología han generado que el tratamiento de esta patología quede 
a discreción del médico.

A pesar del creciente desarrollo de investigación en esta área, se requieren estudios que permitan tener un conocimiento más preciso del mecanismo fisiopatológico subyacente, lo que permitirá un uso racional y ajustado de la terapéutica disponible y la exploración de nuevos fármacos, tanto para el tratamiento sintomático como para la evolución pronóstica.

\section{Bibliografía}

Ache, Y., Guaman, C., Viñole, L., \& Vignolo, G. (01 de 04 de 2020). Infarto agudo de miocardio sin lesiones coronarias obstructivas - MINOCA: un enigma para el cardiólogo clínico. Revista Uruguaya de Cardiología, 35(1). doi:http://dx.doi.org/10.29277/ cardio.35.1.11

Cleves, D., Potosi, J., Gutierrez, J., Mosquera, W., \& Ponce, L. (10 de 06 de 2021). Infarto de miocardio con arterias coronarias no obstruidas en pediatría: una afección poco conocida. Revista Colombiana de Cardiologia, 28(2), 185-188. doi:https://doi. org/10.24875/RCCAR.M21000031

Cohen, H., Iglesias, R., Duronto, R., Lescano, A., Campisi, R., Deviggiano, A., . . Kaski, J. (2021). ISQUEMIA MIOCÁRDICA SIN LESIONES CORONARIAS OBSTRUCTIVAS: MINOCA-INOCA. REVISIÓN PARA LA TOMA DE DECISIONES. Medicina Buenos Aires, 20(3), 253-270. Recuperado el 20 de 10 de 2021, de https://www.medicinabuenosaires.com/indices-de-2020/volumen-80-ano-2020no-3-indice/isquemia/

Corros , C. (05 de 05 de 2021). Sociedad Española de Cardiologia. Recuperado el 24 de 10 de 2021, de https://secardiologia.es/blog/12537-evaluacion-de-los-mecanismos-del-minoca-en-mujeresmediante-uso-combinado-de-tco-y-rmc
Díaz, E. (13 de 11 de 2020). Sociedad Española de Cardiología. Recuperado el 24 de 10 de 2021, de https://secardiologia.es/blog/11977-diagnostico-de-minoca-con-sospecha-de-etiologia-epicardica-ya-esta-claro-el-protocolo

Lozano, H. (08 de 04 de 2021). MINOCA: infarto sin obstrucción coronaria. Portales Medicos, 16(7), 350. Recuperado el 21 de 10 de 2021, de https:// www.revista-portalesmedicos.com/revista-medi$\mathrm{ca} /$ minoca-infarto-sin-obstruccion-coronaria/

Matsudo, M., Aladio, J., Swieszkowski, S., \& Pérez, R. (2019). MINOCA INFARTO CON CORONARIAS NORMALES. Medicina Buenos Aires, 79(3). Recuperado el 19 de 10 de 2021, de https://www.medicinabuenosaires.com/indices-de-2010-a-2019/ volumen-79-ano-2019-no-3-indice/minoca/

Pérez, O. (04 de 08 de 2021). Sociedad Colombiana de Cariologia y Cirguia Cardiovascular. Recuperado el 20 de 10 de 2021, de https://scc.org.co/boletin-no-168-minoca-infarto-del-miocardio-sin-lesiones-obstructivas-un-enfoque-diagnostico/

Tamis, J., Jneid, H., \& Reynolds, H. (04 de 04 de 2019). COLEGIO AMERICANO DE CARDIOLOGIA. Recuperado el 20 de 10 de 2021, de https:// www-acc-org.translate.goog/latest-in-cardiology/ ten-points-to-remember/2019/04/04/15/00/contemporary-diagnosis-and-management-of-patients-with-mi?_x_tr_sl=en\&_x_tr_tl=es\&_x_tr_ $\mathrm{hl}=$ es\&_x_tr_pto=nui,sc

Vidal , R. (17 de 02 de 2017). Sociedad Española de Cardiologia. Recuperado el 20 de 10 de 2021, de https://secardiologia.es/blog/8323-papel-del-tratamiento-medico-en-infarto-de-miocardio-sin-estenosis-coronarias

Zuluaga, M., \& Cano, C. (12 de 2018). Infarto agudo de miocardio sin enfermedad coronaria. Iatreia, 31(4), 371-379. doi:10.17533/udea.iatreia. v31n4a04

\section{CITAR ESTE ARTICULO:}

Soria Bajaña, S. L., De La Pared Ramírez, M. F., Delgado Lazo, K., \& Ortega Romero, N. G. (2021). Infarto agudo al miocardio sin lesiones coronarias obstructivas (MINOCA). RECIMUNDO, 5(4), 163-171. https://doi.org/10.26820/ recimundo/5.(4).oct.2021.163-171

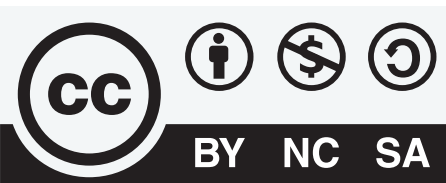

CREATIVE COMMONS RECONOCIMIENTO-NOCOMERCIAL-COMPARTIRIGUAL 4.0. 